Cognition in Context

\title{
Fear not of Cognition in Context
}

\author{
Richard William Prather II \\ University of Maryland, College Park
}

Keywords: cognition; culture, meta-science

Version Date: May 12, 2021

Status: accepted (pending minor revisions) at Infant and Child Development 
I have been working in the study of cognitive development for a little over 20 years. A lot of things have changed, a lot of things have not. I do think there has been genuine progress in many areas of the study of human cognition, developmental or otherwise. And yet, there seems to be a long way to go in characterizing human cognition and its development. We're going to get it together; I believe that.

Now seems like a good time to reflect, not just at my own career but the study of human cognition. Where is the field going? Where has it been? Where ought it go? How do researchers of cognitive development work to make sure that our field evolves such that our contribution to humanity is a positive one; not just edifying but emancipatory? We can take inspiration from researchers near and far. Inspiration may be found in other areas of psychological research (social, cultural, etc.) and other social sciences (public health, sociology, etc.). Frameworks developed in other fields (e.g., Intersectionality, (Crenshaw, 1989) may be helpful for our own interests and questions. If so, this will require some work in determining how these tools can be used to improve the state of cognitive research.

In my career, I've completed studies using a range of approaches and methods. Participants have included college students, children of varying ages, and adults up to 70 years old. My studies have been carried out in a typical lab setting, in K-12 classrooms, and recently online. I've been involved in computational modeling and neuroscience methods in addition to traditional behavioral methods. The most evident thing l've learned is that studying human 
cognition is difficult. Participants' behavior on my tasks often isn't the same in the lab as in the classroom. The surface features of my stimuli seem to affect behavior, at times in unanticipated ways. Results do not always generalize as expected. It can be unclear how well participant samples match samples in the published literature. This can include participant characteristics that are known to be associated with variations in behavior. A study with high socio-economic status (SES) participant sample that makes general conclusions about human cognition, could easily be published in the same journal issue as another study showing that behavior on the same measures vary across SES. While individual studies often appear sound, there has been a nagging feeling of wondering how well my approaches, which fit well within the field norms, contribute to cumulative knowledge of human cognition and development.

Fortunately, I am not the only one wondering about such things. There has been a groundswell of papers focused on scientific reform, meta-science, or advancement of approaches to cognitive research. This work includes the norms around statistical analysis(Dienes, 2011), method registration (Nosek et al., 2018), sharing of data and materials (Martone et al., 2018), large-scale cooperative studies (Frank et al., 2017; Moshontz et al., 2018), participant sample diversity (Roberts et al., 2020; Rowley \& Camacho, 2015) and the relationship between theory and experiments (Devezer et al., 2020; van Rooij \& Baggio, 2020), and other concerns (Borsboom et al., 2021; Ledgerwood, Hudson, et al., 2021; Ledgerwood, Pickett, et al., 2021). Many of these proposals have already had an impact on how we do research. It was not long ago that data sharing, pre-registration, and other aspects of Open Science were rare, if not unheard of. Now, in 2021, they have entered the mainstream, even if 
not the norm. Of course, these proposals have been met with both robust discussion and some feet-dragging from folks who simply don't want to consider changing. I do not bring up the Open Science proposals to suggest that they are "the one way" forward. They are not. I bring

up Open Science to demonstrate that there is clearly a large amount of interest in reconsidering the particulars of how we do science. I lay out my thoughts on how we could reconstruct cognitive research in another piece (Prather, in review). Here, I would like to give room for an important aspect of my standpoint; I contend that, if anything, many reformers do not go far enough in noting that the entire enterprise of behavioral research of human cognition must be reconsidered and reconstructed.

What is the thing that we cognitive researchers are studying? What falls under our scope in characterizing cognition and its development? In addition to receiving typical training in cognition and development in my career, I have sought to learn about other fields. This has often been an informal and somewhat meandering effort, ranging from computational neuroscience to education policy, public health, and sociology. It has been well worth it in contextualizing the effort to understand human cognition and its development. What is being done in other social and behavioral sciences? What tools or frameworks might be of use to cognitive development researchers? What connections currently exist across disciplines, and what connections need to be made? Interdisciplinary work often seems like a good idea but difficult to do. Requires time, resources, and more often than not, collaboration.

One benefit of reading across multiple disciplines is considering differing perspectives. Many fields are concerned to some degree with human behavior; education, sociology, 
psychology, and various combinations thereof. On the surface, researchers in these areas can seem to study similar things, but they often operate based on different fundamental assumptions and what it is they are studying, affecting the questions that are asked, what data is collected, and the nature of the relationship between researcher and participant. There's nothing wrong with these differing perspectives. If anything, it would behoove cognitive researchers to embrace being explicit about their research programs perspective.

Researchers of cognition and development mostly seem interested in connections to biological and natural sciences. I do not criticize wanting to make those connections; they have certainly been useful. However, there's an imbalance in attention to the lower, reductionist levels of analysis. Researchers of basic cognition must embrace interdisciplinarity beyond the occasional neuroimaging collaboration. The levels of analysis "above" us are just as worthy of connection as the ones below. The scope of consideration for cognitive studies must be expanded to understand participants, humans, in context. I do not mean the occasional noncognitive co-variate, such as gender or SES. I mean that our fundamental understanding of what we study must change from an inward-looking focus on internal cognitive processes to an expansive multilevel consideration of humans in their developmental, cultural, and societal context. It is in this context that humans are inextricably embedded. It is in this context that we must position cognition to have any chance of truly understanding it.

Researchers from a sociocultural developmental perspective (Bronfenbrenner, 1977; Rogoff, 2003; Spencer et al., 1997; VygotskiĬ, 1978), certainly have long foregrounded culture in studying human development. The basic contention from this perspective, that humans are imbedded in cultural contexts and should be studied within that context (Rogoff, 2003). For 
researchers who are interested in the "basic" processes of various cognitive processes such as reading, numerical processing and so forth, I do wonder how we can study cognition in a way that considers levels of analysis from the cognitive processes, and the cultural and societal context of participants. How can we balance multiple levels of analysis while avoiding a reductionist approach that priorities low level biological explanations?

\section{The crux of the problem is the pernicious assumption of Cognitive Universalism in our}

approach to studying cognition. The solution is to abandon it. By Cognitive Universalism, I refer to the approach to studying humans that assumes cognitive processes universal to all humans may be characterized by examining a single group of humans with no consideration paid to developmental, cultural, or societal context of that group. I do not think arguing that Cognitive Universalism is a problem is particularly difficult. There are many hints already in the literature (Cheon et al., 2020; Rad et al., 2018). From the WEIRD problem to lack of replication and generality of findings, it's becoming clearer that cognitive universalism is holding back our study of cognition. While these individual issues can be addressed in their own way, they are all, to some degree, part of the foundational structure built in $20^{\text {th }}$-century psychology: the universal structure of cognition. That universal structure has by and large been described by studying high SES white people in the USA. Thus, we have a group of people that represent perhaps $1 \%$ of the global population somehow set as the standard for understanding human cognition and development. Obviously, this is ill-suited, bordering on preposterous. I know that many of my readers understand this, even if implicitly. 


\section{Decades of focus on Cognitive Universalism has led to research norms that make broad} generalizations about humans from homogenous and de-contextualized participant samples.

Norms around child development research broadly may vary across the relevant disciplines

(e.g., Family Science, Human Development). Within the core, basic cognitive research decontextualized lab work remains the norm. Let's say the field's norms may need to be updated. Now what? Research on human cognition and its development must pick itself up and remake itself. This is not simply about the diversity of the participant samples. It is that reevaluating our assumptions about what we are studying, who our participants are, and what we might need to know about them can simultaneously support the theoretical and practical significance of the field if it is to have one.

Can we continue to describe the mental processes of cognition in a way that includes the interaction of biological processes with contextual factors of the person's environment? There some researchers who do so today. Some across social sciences focus on different aspects of the environment, with various human outcomes. Consider educational studies that demonstrate the effects of classroom size, SES, etc., on student grades or other outcomes. These are perfectly legitimate and potentially useful variables to examine. It is an interesting question, but it is not cognition the way we cognitive researchers define it. Here I am differentiating between cognitive tasks as an outcome and examining the underlying processes that may be involved in cognition. On the other hand, we have been trying to study cognitive processes with little connection to environmental influences or outcomes. We may create a great characterization of language learning and tasks that may aid in measuring relevant 
behavior. But that behavior is then measured stripped of any real-world context in order to maintain a controlled experiment, the way we imagine real sciences do it. Developmental, cultural, and societal contexts are relegated to noise to be controlled for, at best, at interesting side variable. It would be one thing if that approach worked. Numerous studies have demonstrated that if the goal is a generalizable characterization of human cognition, ignoring these contextual factors is, in fact, not working well.

Return to the question of what exactly "the thing" is that we study when we study human cognition. The mind might be an answer, or "thinking" or "cognitive processes." All of these sound reasonable enough but, in practice, do not account for the fact that each of these only exists within specific developmental, cultural, and societal contexts that have molded them. As much as we increasingly attend to the measurement of individual behavior in our studies through psychometrics, we must turn equal attention to the measurement of individual context and theorizing about that context. This may mean more than just adding more participant demographic information. Luckily for us, a variety of other social sciences attempt to do this in one way or another. We likely do not need to reinvent the wheel so much as broaden our reading to figure out how our neighbors are making theirs.

Furthermore, if we are looking at these contextual factors, such as SES, we need to be prepared to move beyond the description of the results of systemic inequality in children's context. At some point, we must aim for the root of these inequalities (e.g., racism). This may necessitate work that grapples with broad policy changes. 
A sea-change of sorts may be necessary for those of us interested in human cognition. We must develop an approach that integrates our model of cognition with human context. This will simultaneously improve the verisimilitude of our findings and make connections to applied work much stronger. I believe this is not only a possible goal but one that can soon be accomplished.

\section{References}

Borsboom, D., van der Maas, H. L. J., Dalege, J., Kievit, R. A., \& Haig, B. D. (2021). Theory Construction Methodology: A Practical Framework for Building Theories in Psychology. Perspectives on Psychological Science, 174569162096964. https://doi.org/10.1177/1745691620969647

Bronfenbrenner, U. (1977). Toward an Experimental Ecology of Human Development. American Psychologist, 513-531.

Cheon, B. K., Melani, I., \& Hong, Y. Y. (2020). How USA-Centric Is Psychology? An Archival Study of Implicit Assumptions of Generalizability of Findings to Human Nature Based on Origins of Study Samples. Social Psychological and Personality Science, 11(7), 928-937. https://doi.org/10.1177/1948550620927269

Crenshaw, K. (1989). Demarginalizing the Intersection of Race and Sex: A Black Feminist Critique of Antidiscrimination Doctrine, Feminist Theory and Antiracist Politics. University of Chicago LEgal Forum, 1989(1), 139-167. https://doi.org/10.4324/9781315631011-38

Devezer, B., Navarro, D. J., Vandekerckhove, J., \& Buzbas, E. O. (2020). The case for formal methodology in scientific reform [Preprint]. Scientific Communication and Education. https://doi.org/10.1101/2020.04.26.048306

Dienes, Z. (2011). Bayesian Versus Orthodox Statistics: Which Side Are You On? Perspectives on Psychological Science, 6(3), 274-290. https://doi.org/10.1177/1745691611406920

Frank, M. C., Bergelson, E., Bergmann, C., Cristia, A., Floccia, C., Gervain, J., Hamlin, J. K., Hannon, E. E., Kline, M., Levelt, C., Lew-Williams, C., Nazzi, T., Panneton, R., Rabagliati, H., Soderstrom, M., Sullivan, J., Waxman, S., \& Yurovsky, D. (2017). A Collaborative Approach to Infant Research: Promoting Reproducibility, Best Practices, and TheoryBuilding. Infancy, 22(4), 421-435. https://doi.org/10.1111/infa.12182

Ledgerwood, A., Hudson, S. T. J., Lewis, N. A., Maddox, K. B., Pickett, C., Remedios, J. D., Cheryan, S., Diekman, A., Dutra, N. B., Goh, J. X., Goodwin, S., Munakata, Y., Navarro, D., Onyeador, I. N., Srivastava, S., \& Wilkins, C. L. (2021). The Pandemic as a Portal: Reimagining Psychological Science as Truly Open and Inclusive [Preprint]. PsyArXiv. https://doi.org/10.31234/osf.io/gdzue

Ledgerwood, A., Pickett, C., Navarro, D., Remedios, J. D., \& Lewis, N. A. (2021). The Unbearable Limitations of Solo Science: Team Science as a Path for more Rigorous and Relevant Research [Preprint]. PsyArXiv. https://doi.org/10.31234/osf.io/5yfmq 
Martone, M. E., Garcia-Castro, A., \& VandenBos, G. R. (2018). Data sharing in psychology. American Psychologist, 73(2), 111-125. https://doi.org/10.1037/amp0000242

Moshontz, H., Campbell, L., Ebersole, C. R., IJzerman, H., Urry, H. L., Forscher, P. S., Grahe, J. E., McCarthy, R. J., Musser, E. D., Antfolk, J., Castille, C. M., Evans, T. R., Fiedler, S., Flake, J. K., Forero, D. A., Janssen, S. M. J., Keene, J. R., Protzko, J., Aczel, B., ... Chartier, C. R. (2018). The Psychological Science Accelerator: Advancing Psychology Through a Distributed Collaborative Network. Advances in Methods and Practices in Psychological Sciences, 1(4), 15.

Nosek, B. A., Ebersole, C. R., DeHaven, A. C., \& Mellor, D. T. (2018). The preregistration revolution. Proceedings of the National Academy of Sciences, 115(11), 2600-2606. https://doi.org/10.1073/pnas.1708274114

Prather, R. W. (in review). Reconstructing the study of Human Cognition.

Rad, M. S., Martingano, A. J., \& Ginges, J. (2018). Toward a psychology of Homo sapiens: Making psychological science more representative of the human population. Proceedings of the National Academy of Sciences of the United States of America, 115(45), 11401-11405. https://doi.org/10.1073/pnas.1721165115

Roberts, S. O., Bareket-Shavit, C., Dollins, F. A., Goldie, P. D., \& Mortenson, E. (2020). Racial Inequality in Psychological Research: Trends of the Past and Recommendations for the Future. Perspectives on Psychological Science, 15(6), 1295-1309. https://doi.org/10.1177/1745691620927709

Rogoff, B. (2003). The cultural nature of human development. Oxford University Press. Rowley, S. J., \& Camacho, T. C. (2015). Increasing Diversity in Cognitive Developmental Research: Issues and Solutions. Journal of Cognition and Development, 16(5), 683-692. https://doi.org/10.1080/15248372.2014.976224

Spencer, M. B., Dupree, D., \& Hartmann, T. (1997). A Phenomenological Variant of Ecological Systems Theory (PVEST): A self-organization perspective in context. Development and Psychopathology, 9(4), 817-833. https://doi.org/10.1017/S0954579497001454

van Rooij, I., \& Baggio, G. (2020). Theory Before the Test: How to Build High-Verisimilitude Explanatory Theories in Psychological Science. 16.

Vygotskiĭ, L. S. (1978). Mind in society: The development of higher psychological processes (M. Cole \& Vera John-Steiner, Sylvia Scribner, Ellen Souberman, Eds.). Harvard University Press. 